\title{
VigILÂNCIA EM SAÚde: É NeCESSÁRIA UMA LEGISLAÇão DE EMERGÊNCIA?
}

\author{
HEALTH SURVEILLANCE: \\ IS IT NECESSARY AN EMERGENCY LEGISLATION?
}

\author{
Maria Glória Teixeira ${ }^{(*)}$ \\ Maria da Conceição N. Costa ${ }^{(* *)}$ \\ Itana Viana ${ }^{(* * *)}$ \\ Jairnilson Silva Paim ${ }^{(* * * *)}$
}

\section{RESUMO}

A Lei n. 8.080/1990 que rege o Sistema Único de Saúde (SUS) inclui no seu texto a Vigilância Epidemiológica/VE, ampliando o seu conceito. Todavia, legalmente este campo da saúde pública permanece subordinado à Lei $\mathrm{n}$. 6.259/1975. Projetos de Lei vêm sendo elaborados para substituir esta última; porém, não estão em consonância com a Lei n. 8.080. Ademais, a promulgação do novo Regulamento Sanitário Internacional (RSI/2005) exige que o país elabore instrumento legal para atender às exigências desse Código Sanitário. Este artigo tem como objetivo apresentar algumas reflexões sobre os Anteprojetos de Lei apresentados e possíveis repercussões no processo de consolidação dos princípios que orientam a Reforma Sanitária Brasileira. Descreve-se a criação e desenvolvimento do Sistema Nacional de Vigilância Epidemiológica no Brasil e destaca-se que a Lei à qual a VE está subordinada, ainda que promulgada em plena Ditadura Militar, não previa sanções aos indivíduos. No entanto, os referidos Anteprojetos contemplam a instituição de infrações e crimes sanitários, fato rechaçado pela comunidade que milita no campo da Saúde Coletiva, por entender que tais dispositivos são autoritários, invasivos à liberdade individual e violam direitos que asseguram a dignidade da pessoa humana. Caso necessário, recomenda-se que uma

(*) Professor do Instituto de Saúde Coletiva da Universidade Federal da Bahia (UFBA). E-mail: $<$ magloria@ufba.br>. Recebido em 20.05.09. Aprovado em 30.06.09.

$\left({ }^{* *}\right)$ Professor do Instituto de Saúde Coletiva da UFBA.

$\left.{ }^{* * *}\right)$ Promotora de Justiça do Ministério Público do Estado da Bahia.

$\left.{ }^{\star \star * *}\right)$ Professor do Instituto de Saúde Coletiva da UFBA. 
nova Lei para o SNVS seja consentânea com o Estado Democrático de Direito e com princípios da Reforma Sanitária Brasileira e do SUS. Ressalta-se que medidas restritivas sejam transitórias e cuidadosamente elaboradas, para que, em nome da necessidade do uso de força para controle de riscos à saúde, atos arbitrários não venham a ser cometidos pelas autoridades.

\section{Palavras-chave}

Direito Sanitário; Emergências em Saúde Pública; Lei de Vigilância; Vigilância em Saúde; Reforma Sanitária.

\section{ABSTRACT}

The Law 8.080/90 which regulates the Brazilian National Public Health System includes the Epidemiologic Surveillance, expanding the concept. However legally this field of public health remains subject to the Law 6.259/ 1975. Bills are being drafted to replace the latter, but they are not in accordance to the 8.080 Act. Morever the promulgation of the new International Health Regulations (RSI/2005) requires that the country elaborates legal instrument to meet the demands of the International Code. This article aims to present some reflections on the preliminaries drafts of laws presented and their possible implications in the process of consolidation of principles that guide the Brazilian Sanitary Reform. It describes the creation and development of the National System of Epidemiological Surveillance in Brazil, highlighting the Law to which the Epidemiologic Surveillance is subject. Moreover, this Law has been promulgated during the military dictatorship it did not mention punishments against individuals. However, these preliminaries drafts of projects include the establishment of health violations and crimes which is rejected by the community involved with the field of Public Health, once this community believes that such devices are authoritarians, restraints of the individual freedom and that they violate rights such as the human dignity. If it is necessary, it is recommended that a new Law for the National System of Epidemiological Surveillance should be in accordance to the Rule of Law and democratic principles of the Brazilian Health Reform and the Brazilian National Public Health System. It is emphasized that restrictive measures should be transitor and carefully elaborate to avoid arbitrary acts from authorities, on behalf of the necessity of using force to control risks to health.

\section{Keywords}

Health Law; Health Surveillance; Public Health Emergencies; Sanitary Reform; Surveillance Law. 


\section{INTRODUÇÃO}

Desde o Brasil colônia, o poder público tem estabelecido uma série de ações e criado um conjunto de estruturas para responder aos problemas sanitários da população. Apesar de muitas políticas de saúde, neste país, serem decorrentes de interesses econômicos, reconhece-se que essas iniciativas também têm procurado se pautar em informações e conhecimentos científicos oriundos das áreas biomédicas e da Saúde Pública.

No intuito de enfrentar problemas de saúde da população, o Brasil implantou uma organização sanitária centrada na dicotomia entre a assistência médica e a saúde pública. Esta última se desenvolvia em dois campos de atuação: um deles, a Vigilância Sanitária, cuja prática vem sendo calcada em legislações, normas e padrões ${ }^{(1)}$ e é voltada para ações de controle sanitário (defesa e proteção do meio ambiente, produção e circulação de bens de consumo, prestação de serviços de interesse para a saúde dos cidadãos); o segundo, a Vigilância e Controle de Doenças, teve sua origem nas intervenções para conter as doenças pestilenciais prevalentes no início da nossa colonização, que culminou na instituição das grandes campanhas de erradicação e/ou controle de doenças nos primeiros anos do século XX.(2)

Todavia, a partir do delineamento, em 1986, do ideário da Reforma Sanitária Brasileira explicitado na $8^{a}$ Conferência Nacional de Saúde vêm sendo discutidas propostas de modelos assistenciais que, coerentes com os princípios do Sistema Único de Saúde (SUS), sejam capazes de articular ações de promoção, prevenção, recuperação e reabilitação nas suas dimensões individuais e coletivas $^{(3)}$, visando à atenção integral à saúde da população.

No bojo dessas discussões, incluiu-se a necessidade de reformulação e fortalecimento da área de Vigilância Epidemiológica e Controle de Doenças mediante o desenvolvimento e/ou aperfeiçoamento técnico-metodológico para o diagnóstico das condições de vida e saúde das populações, a ser apropriado pelos sistemas locais de saúde. A proposta era avançar na direção da elaboração e implantação de modelos de atenção, na perspectiva da melhoria da qualidade da assistência individual e coletiva, e resolução de problemas de saúde de grupos populacionais( ${ }^{(4)}$, sob a égide dos princípios

(1) COSTA, E. A. Vigilância sanitária, defesa e proteção da saúde. 1998. 350 p. Tese (Doutorado) - Faculdade de Saúde Pública, Universidade de São Paulo, 1998.

(2) Id. Ibid.; COSTA, Nilson do Rosário. Lutas urbanas e controle sanitário: origens das políticas de saúde no Brasil. Rio de Janeiro: Vozes, 1985; ESCOREL, S.; TEIXEIRA, L. A. Historia das políticas de saúde no Brasil de 1822 a 1963: do império ao desenvolvimento populista. In: GIOVANELLA, L. (Org.). Políticas e sistemas de saúde no Brasil. Rio de Janeiro: Fiocruz, 2008. p. 333-384.

(3) TEIXEIRA, C. F.; PAIM, J. S.; VILASBÔAS, A. L. SUS, modelos assistenciais e vigilância da saúde. Inf. Epidemiol. SUS, v. 7, n. 2, p. 7-28, abr./jun. 1998.

(4) Id. Ibid.; PAIM, J. S.; TEIXEIRA, M. G. Reorganização do sistema de vigilância epidemiológica na perspectiva do Sistema Único de Saúde - SUS. Inf. Epidemiol, SUS, v. 1, n. 5, p. 27-57, out. 1992. 
constitucionais e da Lei Orgânica da Saúde, dentre os quais se destacam a universalização do cuidado, descentralização das ações e atenção integral à saúde da população.

Ao lado deste movimento de reformas na saúde, de lutas pela redemocratização do país e da dinâmica da conjuntura política e social do mundo globalizado e, principalmente, após os acontecimentos de 11 de setembro de 2001, no cenário internacional também foi observado outro movimento visando ao fortalecimento de sistemas nacionais de vigilância epidemiológica. Este contexto influenciou nas definições das modificações do novo Regulamento Sanitário Internacional (RSI), aprovado em 2005 pelos Países-Membros da Organização Mundial da Saúde (OMS), que estabeleceu mecanismos de controle governamentais para as situações consideradas como "Emergências de Saúde Pública de interesse internacional", ou seja, aquelas que supostamente colocam sob risco a saúde e a segurança das nações do ponto de vista sanitário.

O presente artigo tem como objetivo apresentar algumas reflexões sobre estas últimas iniciativas e possíveis repercussões no processo de consolidação dos princípios democráticos brasileiros, particularmente os que orientam o processo da Reforma Sanitária Brasileira e do SUS, em particular, no que concerne às ações da Vigilância Epidemiológica.

\section{ORIGEM DA VIGILÂNCIA EPIDEMIOLÓGICA}

$\mathrm{Na}$ área da saúde, o termo vigilância nasceu com as práticas de quarentena que correspondiam ao conjunto de atividades de observação sistemática e ativa de indivíduos suspeitos de serem portadores de doenças transmissíveis. Na década de 1950, quando as grandes campanhas sanitárias dirigidas ao combate de enfermidades específicas estavam alcançando redução substantiva de suas incidências, a expressão "vigilância epidemiológica" passou a designar uma das etapas constitutiva de algumas campanhas de erradicação de doenças, a exemplo da malária, febre amarela e varíola(5).

A partir de então, concepções mais amplas de vigilância, com enfoque populacional, foram estabelecidas abrangendo doenças transmissíveis e não transmissíveis ${ }^{(6)}$. As ideias, debates, e experiências práticas geradas no

(5) LANGMUIR, A. D. The surveillance of communicable diseases of national importance. New Engl J Med, n. 268, p. 182-192, 1963; FOSSAERT, D. H.; LLOPIS, A.; TIGRE, C. H. Sistemas de vigilância epidemiológica. Bol. Ofic. Sanit. Panam., n. 76, p. 512-525, 1974; TEIXEIRA, M. G.; RISI JÚNIOR, J. B.; COSTA, M. C. N. Vigilância epidemiológica. In: ROUQUAYROL, M. Z.; ALMEIDA FILHO, N. Epidemiologia e saúde. 6. ed. Rio de Janeiro: Medsi, 2003. p. 313-356.

(6) LANGMUIR, A. D. op. cit.; RASKA, K. National and international surveillance of communicable diseases. Who Chron, n. 20, p. 31-21, 1966. 
período permitiram construir as bases conceituais e metodológicas dos sistemas de vigilância epidemiológica instituídos nas décadas seguintes, em muitos países ocidentais.

No cenário internacional, a incorporação dos princípios técnico-científicos para a implantação dos Sistemas de Vigilância Epidemiológica se deu, em 1965, quando foi criada a Unidade de Vigilância Epidemiológica (UVE) da OMS. Ao final dos anos $1960^{(7)}$, foi revisado o primeiro Regulamento Sanitário Internacional formulado em 1951, ocasião em que foram excluídas algumas das doenças com obrigatoriedade de notificação internacional. Foram mantidos no novo documento os mecanismos de notificação compulsória de doenças de interesse internacional à época (febre amarela, varíola, peste e cólera), bem como os princípios de máxima proteção contra a propagação de enfermidades entre países e de mínima restrição para as viagens e comércio internacional estabelecidos, desde 1851, na Primeira Conferência Sanitária Internacional realizada em Veneza.

\section{A VIGILÂNCIA EPIDEMIOLÓGICA NO BRASIL}

O Sistema Nacional de Vigilância Epidemiológica (SNVE) foi criado em 1975, por meio da Lei n. 6.259 que dispunha sobre a organização das ações de vigilância epidemiológica entendida como “... informações, investigações e levantamentos necessários à programação e à avaliação das medidas de controle de doenças e de situações de agravos à saúde". Esta iniciativa ocorreu impulsionada pelo clamor da sociedade para que 0 governo adotasse medidas substantivas para melhoria da assistência médica e ações de controle voltadas para enfrentamento da epidemia de meningite meningocócica que assolava o país, desde 1972.

Até a primeira metade da década de 1990, as informações e ações desenvolvidas pelo SNVE implantado no país quase não contemplavam o nível municipal(8), embora tenha sido concebido para se estruturar em um modelo hierárquico de responsabilidade, desenvolvendo quatro níveis de gestão (Ministério da Saúde, Secretarias Estaduais de Saúde, Diretorias Regionais de Saúde e Unidades Locais de Vigilância Epidemiológica) ${ }^{(9)}$.

Apesar de o SNVE ter sido inspirado sob um modelo verticalizado, suas atividades foram sendo estruturadas e passaram a ser desenvolvidas

(7) ORGANIZAÇÃO MUNDIAL DA SAÚDE. Regulamento Sanitário Internacional, 1969. 3. ed. anot. Ginebra: OMS, 1983.

(8) PAIM, J. S.; TEIXEIRA, M. G. op. cit., p. 25-57.

(9) BRASIL. Decreto n. 78.231 de 12 de agosto de 1976. Regulamenta a Lei n. 6.259, de 30 de outubro de 1975, que dispõe sobre a organização das ações de Vigilância Epidemiológica, sobre o Programa Nacional de Imunizações, estabelece normas relativas à notificação compulsória de doenças, e dá outras providências. Disponível em: <http://www.aids.gov.br/c-geral//c0303.htm>. Acesso em: 15 abr. 2009. 
em todos os estados da federação, os quais também eram responsáveis pelas ações de vigilância nos municípios. À época, esta vigilância era direcionada para doenças infecciosas e parasitárias selecionadas (notificação compulsória) cuja relação, obrigatoriamente, incluía aquelas listadas no RSI/ 1969 , outras consideradas de interesse nacional definidas de acordo com critérios técnicos preestabelecidos, além de "casos de agravos inusitados à saúde" e situações de calamidade pública. Cabia às instituições de saúde federais e estaduais, por meio da estruturação de órgãos de vigilância e controle, o desenvolvimento de mecanismos e ações de prevenção, controle e contenção desses problemas ${ }^{(10)}$. Observe-se que a notificação obrigatória das doenças de interesse internacional, pelo Ministério da Saúde do Brasil à OPAS/OMS, somente era feita após confirmação diagnóstica dos casos e óbitos.

No que se referia às obrigações individuais, a Lei n. 6.259/1975 instituiu como dever de todo cidadão "... comunicar à autoridade sanitária local a ocorrência de fato, comprovado ou presumível, de caso de doença transmissível ... ". A obrigatoriedade da notificação de casos suspeitos ou confirmados das doenças incluídas na lista de notificação compulsória cabia a "médicos e outros profissionais de saúde no exercício da profissão, bem como aos responsáveis por organizações e estabelecimentos públicos e particulares de saúde e ensino...”. Esta Lei, embora promulgada em plena ditadura militar instituída em 1964, não previa sanções aos indivíduos, garantindo a confidencialidade da notificação dos casos, salvo em situações excepcionais de "grande risco à comunidade a juízo da autoridade sanitária e com conhecimento prévio do paciente ou do seu responsável". Mesmo sob o regime autoritário, este instrumento legal não estabelecia a priori intervenções restritivas a indivíduos ou grupos populacionais. As medidas de prevenção e controle competiam às autoridades sanitárias, de acordo com cada situação específica, considerando o conhecimento técnico-científico vigente.

\section{O NOVO REGULAMENTO SANITÁRIO INTERNACIONAL}

A revisão do Regulamento Sanitário Internacional de 1969 foi realizada entre 1995 e 2005. Diante do atual contexto de intenso tráfico internacional do mundo globalizado e das mudanças epidemiológicas, a resolução da Assembleia Mundial de Saúde (1995) que decidiu proceder a esta revisão também recomendou, dentre outras orientações, que esta contemplasse

(10) BRASIL. Decreto n. 78.231, de 12 de agosto de 1976. Regulamenta a Lei n. 6.259, de 30 de outubro de 1975, que dispõe sobre a organização das ações de Vigilância Epidemiológica, sobre o Programa Nacional de Imunizações, estabelece normas relativas à notificação compulsória de doenças, e dá outras providências. Disponível em: <http://www.aids.gov.br/c-geral/lc0303.htm>. Acesso em: 15 abr. 2009. 
novas estratégias de vigilância e controle de enfermidades transmissíveis, que não estivessem restritas a uma reduzida lista de notificação compulsória e que incluísse outros eventos inusitados de saúde (produzidos por agentes não biológicos) com potencial de colocar sob risco a saúde das populações dos países.

A ocorrência de doenças emergentes e reemergentes foi um dos temas que permeou as discussões dos trabalhos técnicos da revisão desse Código Sanitário, de modo que, logo nos primeiros anos, foi proposta a notificação mais precoce de entidades clínicas suspeitas visando obter maior agilidade na coleta de amostras biológicas para o diagnóstico laboratorial (de novos agentes). Inicialmente supôs-se que a substituição de notificação internacional para doenças específicas por síndromes (febres hemorrágicas, respiratórias agudas, gastrointestinais incluindo as síndromes diarreicas agudas e as síndromes ictéricas agudas, neurológicas agudas e outras mal definidas) poderia atender ao propósito de agilização da notificação de possíveis novos agentes e mesmo dos reemergentes. Felizmente, para fundamentar e testar a viabilidade desta proposta estudos pilotos foram conduzidos em alguns países, sob os auspícios da OMS. As análises de seus resultados apontaram a inviabilidade técnico-científica e operacional dessa proposição, em virtude da elevada sensibilidade e baixa especificidade, o que acarretaria uma sobrecarga para os sistemas nacionais de vigilância e também para as regionais da OMS, desde quando uma vastidão de entidades clínicas passaria a ser exaustivamente notificada antes de se proceder e concluir as investigações epidemiológicas e clínico-laboratoriais que viessem indicar, com algum grau de certeza, a existência de riscos populacionais à saúde.

Essa proposta foi abandonada e, após alguns anos de debates dos grupos de trabalho, foi aprovado o Novo Regulamento Sanitário Internacional na Assembleia Mundial de Saúde de $2005^{(11)}$ trazendo modificações substantivas no capítulo que se refere à vigilância de doenças e riscos, dentre as quais se inclui a exigência de notificação das denominadas "Emergências de Saúde Pública de Importância Internacional"(12), definidas como eventos extraordinários determinados por: a) constituir um risco de saúde pública para outro estado por meio da propagação internacional de doenças; b) requerer, potencialmente, uma resposta internacional coordenada.

Um "algoritmo de decisão" foi desenvolvido para facilitar a operacionalização da noção de emergência de interesse internacional (RSI/2005). Neste código, "Evento" é definido como a manifestação de uma doença ou uma ocorrência que cria um potencial para uma doença, o que significa que esta

(11) WORLD HEALTH ASSEMBLY. Revision of the International Health Regulations, WHA 58.3. 2005. Disponível em: <http://www.who.int>. Acesso em: 10 maio 2009.

(12) Id. Ibid. 
concepção foi ampliada na medida em que além de doenças manifestas, fatores de risco também foram considerados.

\section{A VIGILÂNCIA EPIDEMIOLÓGICA NO PROCESSO DE REDEMOCRATIZAÇÃO DO BRASIL}

Com a promulgação da Constituição de $1988^{(13)}$, foi formulado um conceito ampliado de saúde e de seus determinantes ao lado da criação de um Sistema Único de Saúde (SUS). Dentre outros princípios e diretrizes, este Sistema incluiu a descentralização e a integralidade da atenção à saúde, e reconheceu formalmente a Vigilância Epidemiológica ${ }^{(14)}$, fato que contribuiu para o fortalecimento e aprimoramento dessa área em todo o país.

Para atender às responsabilidades do nível central do SUS, definidas na Lei Orgânica da Saúde ${ }^{(15)}$ no que se refere a este campo, foi criado, em 1991, o Centro Nacional de Epidemiologia/CENEPI, como

instância nacional com a competência de promover e disseminar o uso da metodologia epidemiológica em todos os níveis do SUS para subsidiar a formulação e a implementação de políticas, bem como a organização dos serviços e ações de saúde.

A partir de então, o CENEPI passou a estimular o processo de descentralização das ações de vigilância epidemiológica e controle de doenças, definindo junto aos Conselhos de Secretários Municipais e Estaduais (CONASS, CONASEMS), e na Comissão Tripartite, o desenvolvimento de critérios (epidemiológicos, geográficos, populacionais, dentre outros) mais equânimes para distribuição dos recursos orçamentários e financeiros destinados às ações de vigilância e controle de doenças e à criação de novos instrumentos organizacionais e normativos, indicadores epidemiológicos de avaliação, responsabilidades compartilhadas, dentre outras iniciativas.

Todo este processo foi conduzido tendo como marco não só a materialização da proposta de descentralização e universalização da atenção à saúde, um dos eixos centrais da Reforma Sanitária Brasileira, como também o respeito ao princípio constitucional(16) que estabelece que ninguém

(13) BRASIL. Constituição da República Federativa do Brasil: promulgada em 5 de outubro 1988. São Paulo. Saraiva, 2000.

(14) BRASIL. Ministério da Saúde. Lei n. 8.080 de 19 de setembro de 1990: dispõe sobre as condições para promoção, proteção e recuperação da saúde, a organização e o funcionamento dos serviços correspondentes e dá outras providências. Brasília: Ministério da Saúde, 1990. Disponível em: <http://www6.senado.gov.br/legislacao/ListaPublicacoes.action?id=134238>. Acesso em: 30 abr. 2009.

(15) Id. Ibid.

(16) BRASIL. Ministério da Saúde. Conferência Nacional de Saúde, 11. Caderno ... Brasília: Ministério da Saúde, 2000. 
será obrigado a fazer ou deixar de fazer alguma coisa que a lei não obrigue. Observa-se assim, que mudanças significativas ocorreram no Sistema de Vigilância Epidemiológica do país nos últimos 15 anos, principalmente direcionados para o fortalecimento da execução de ações de vigilância e controle de doença em cada espaço geográfico. São exemplos concretos, a informatização e implantação, nos municípios, de sistemas de informação de base epidemiológica, repasses regulares de recursos financeiros, capacitação de recursos humanos para o desenvolvimento das atividades inerentes a esta função essencial de saúde pública, entre outros.

Estes fatos demonstram que, ainda que a Vigilância Epidemiológica e Controle de Doenças no Brasil acumule certas deficiências, avanços significativos têm sido obtidos nos últimos 20 anos que vêm se refletindo na situação de saúde da população, como, por exemplo, na redução da mortalidade por diarreias e infecções respiratórias agudas, eliminação do sarampo e controle do tétano neonatal, entre outros, que contribuíram para a mudança do padrão de mortalidade por doenças infecciosas e parasitárias (DIP), embora, ainda se conviva com um complexo padrão de ocorrência das doenças infectocontagiosas onde se observa muitas enfermidades com tendência declinante e outras com quadro de persistência. ${ }^{(17)}$

Ademais, a emergência de novas e reemergência de antigas doenças infecciosas vêm desafiando os sistemas de saúde ${ }^{(18)}$, não só de países em desenvolvimento como o Brasil, como também dos países desenvolvidos, razão pela qual a preocupação com os problemas de saúde decorrentes das doenças transmissíveis voltou ao centro das agendas dos países centrais, visto que, muitas vezes, a ocorrência destas doenças resulta em situações de epidemias e agravos inusitados, que são considerados ameaçadores do ponto de vista internacional. ${ }^{(19)}$

Conforme descrito, paralelamente ao processo de construção de consenso entre os Países-Membros da OMS para promulgação do novo RSI, a Vigilância Epidemiológica no Brasil foi se estruturando para enfrentar e conter endemias e epidemias que ocorrem em seu território, tendo em vista, que com a implantação e fortalecimento do SUS, recursos humanos vêm sendo capacitados em larga escala, ao lado de contínuo processo de modernização da estrutura dos sistemas nacional, estaduais e locais de Vigilância.

(17) CARMO, E. H.; BARRETO, M. L.; SILVA JUNIOR, J. B. Mudanças nos padrões de morbimortalidade da população brasileira: os desafios para um novo século. Epidemiol. Serv. Saúde, v. 12, n. 2, p. 6375, abr./jun. 2003.

(18) BARATA, R. C. B. O desafio das doenças emergentes e a revalorizaçäo da epidemiologia descritiva. Revista de Saúde Pública, São Paulo, v. 31, n. 5, p. 531-537, 1997.

(19) BARRETO, M. L. Determinantes das condições de saúde e problemas prioritários do país. In: Conferência Nacional de Saúde, 11. Caderno... Brasília: Ministério da Saúde; 2000. p. 235-259; CARMO, E. H.; PENNA, G.; OLIVEIRA, W. K. Emergências de saúde pública: conceito, caracterização, preparação e resposta. Estudos Avançados, São Paulo, v. 22, n. 64, p. 19-32, 2008. 
Além disso, foram implantadas Salas de Situação (Centro de Informações Estratégicas de Vigilância em Saúde/CIEVS), nos três níveis de gestão do SUS, dispondo de profissionais capacitados que monitoram continuamente e alertam, quando pertinente, para a necessidade imediata de investigações e medidas de contenção desses eventos ${ }^{(20)}$, o que permite ágil mobilização de recursos com vistas ao desencadeamento das medidas indicadas.

A principal exceção à efetividade do sistema de vigilância se refere ao controle da dengue, doença que também vem vitimando a população em mais de 70 países do mundo subdesenvolvido e desenvolvido. Esta situação se mantém não só devido às dificuldades operacionais que o sistema brasileiro vem enfrentando para desenvolver as atividades preconizadas para o combate vetorial em todos os municípios infestados pelo Aedes aegypti, como também, em virtude das insuficiências técnico-científicas que ainda persistem no que se refere à prevenção e tratamento desta virose, cujo agente vem exibindo extraordinária força de transmissão sem responder, no mais das vezes, às medidas de controle disponíveis. ${ }^{(21)}$

\section{AS PROPOSTAS DE PROJETOS DE LEI PARA O SISTEMA NACIONAL DE VIGILÂNCIA EM SAÚDE E SITUAÇÕES DE EMERGÊNCIAS DE SAÚDE PÚBLICA}

Na medida em que até o presente momento ainda não foi aprovado nenhum instrumento específico em substituição à Lei n. 6.259 de 1975, a ampliação do escopo de atuação e as reformulações normativas que estão sendo imprimidas ao SNVS têm se baseado nos princípios e diretrizes do SUS ${ }^{(22)}$. No entanto, formalmente este sistema permanece subordinado àquele instrumento legal.

Em razão deste descompasso, desde os primeiros anos deste século, alguns Anteprojetos de Lei vêm sendo elaborados por técnicos do Ministério da Saúde (MS) com vistas à formalização do Sistema Nacional de Vigilância em Saúde (neste contexto entendido como somatório das vigilâncias epide-

(20) TEIXEIRA, M. G.; COSTA, M. C. N. Vigilância epidemiológica: políticas, sistemas e serviços. In: GIOVANELLA, L. (Org.). Políticas e sistemas de saúde no Brasil. Rio de Janeiro: Fiocruz, 2008. p. 795-817; CARMO, E. H.; PENNA, G.; OLIVEIRA, W. K. op. cit.

(21) GUBLER, D. J. Dengue and dengue hemorrhagic fever. Clin. Microbiol. Rev., v. 11, n. 3, p. 480496, 1998; OOI, E. E.; GOH, K. T.; GUBLER, D. J. Dengue Prevention and 35 Years of Vector Control in Singapore. Emerg Infect Dis, v. 12, n. 6, p. 887-893, 2006; TEIXEIRA, M. G.; COSTA, M. C. N.; BARRETO, F. Dengue: twenty-five years since reemergence in Brazil. Cad. Saúde Pública, v. 25, suppl. 1, p. S7-S18, 2009.

(22) BRASIL. Ministério da Saúde. Lei n. 8.080, de 19 de setembro de 1990: dispõe sobre as condições para promoção, proteção e recuperação da saúde, a organização e o funcionamento dos serviços correspondentes e dá outras providencias, cit. 
miológica, ambiental e de saúde do trabalhador), no propósito, segundo o MS, de criar um "ambiente jurídico-institucional necessário para que o Brasil enfrente os desafios sanitários deste novo século..."(23)

O primeiro Projeto de Lei, datado de 2002, propunha a criação de uma Agência Federal de Prevenção e Controle de Doenças - (APEC) (que seria sucedânea do CENEPI) e, em um dos seus capítulos, sob o título "Da Emergência Epidemiológica", apresentava proposições legais que se assemeIhavam àquelas que balizaram a atuação da polícia sanitária do Brasil no início do século $X X^{(24)}$. De acordo com aquele projeto, o Ministro de Estado da Saúde, por proposta do Presidente da APEC, poderia estabelecer "Estado de Quarentena Federal", dispondo "sobre o isolamento de indivíduos, animais e comunidades em situação de risco" e a interdição de ambientes ou meios de transporte, além de determinar o acompanhamento médico de indivíduos e a necessidade destes se reportarem, periodicamente, à "autoridade de epidemiologia".

Afortunadamente, a proposta da Agência não floresceu e, em substituição ao CENEPI, foi criada, em 2003, a Secretaria de Vigilância em Saúde (SVS/MS), ato considerado um avanço para o desenvolvimento do SUS ${ }^{(25)}$, em razão de ter sido inserida na estrutura central do Ministério da Saúde no mesmo patamar hierárquico da Secretaria responsável pela área de assistência médica individual. Este fato poderia ser entendido como um primeiro passo no sentido da superação da dicotomia do modelo assistencial vigente, sinalizando para a possibilidade de construção de modelos de atenção "voltados para a qualidade, efetividade, equidade e necessidades prioritárias de saúde", conforme proposto pela 11르 Conferência Nacional de Saúde.(26)

Em 2006, a discussão de outro Anteprojeto de Lei, elaborado pela SVS/MS, constituiu-se em tema de uma Oficina de Trabalho da 6 ${ }^{\underline{a}}$ Expoepi Mostra Nacional de Experiências Bem-Sucedidas em Epidemiologia(27), com vistas à formalização do Sistema Nacional de Vigilância em Saúde (SINVES),

(23) BRASIL. Ministério da Saúde. Secretaria de Vigilância em Saúde. Projeto de Lei do Sistema Nacional de Vigilância em Saúde. Brasília: SVS/CEPEDISA/USP, 2006. (Texto para uso interno na oficina da 6 $6^{\mathrm{a}}$ EXPOEPI, realizada em Brasília em 14 nov. 2006). Disponível em: <http:// www.anerbrasil.org.br/sites/700/797/00000101.doc.>. Acesso em: 6 maio 2009.

(24) BRASIL. Projeto de Lei. Dispõe sobre os Sistemas Nacionais de Epidemiologia, de Saúde Ambiental e de Saúde Indígena, cria a Agência Federal de Prevenção e Controle de Doenças APEC, e dá outras providências. 2002. Disponível em: <www.camara.gov.br/sileg/ MostrarIntegra.asp?CodTeor=49706>. Acesso em: 15 abr. 2009; EDITORIAL. Outra emenda pior que o soneto: APEC - novas ameaças ao processo de descentralização da saúde. Bol Abrasco, n. 84, 2002.

(25) TEIXEIRA, M. G. Editorial especial: Secretaria de Vigilância em Saúde: novas perspectivas para o SUS. Rev. Bras. Epidemiol., v. 6, n. 3, p. 197-199, 2003.

(26) BARRETO, M. L. Determinantes das condições de saúde e problemas prioritários do país, cit. (27) BRASIL. Ministério da Saúde. Secretaria de Vigilância em Saúde. Projeto de Lei do Sistema Nacional de Vigilância em Saúde. Brasília: SVS/CEPEDISA/USP, 2006, cit. 
ocasião em que participaram representantes do CONASS, CONASEMS, profissionais que atuam nos órgãos de epidemiologia do SUS das três esferas de governo, instituições acadêmicas e associações médicas e de saúde coletiva. Este Anteprojeto propunha estabelecer “... instrumentos e medidas de prevenção e controle de doenças e de riscos e agravos à saúde no Brasil" e, em seu caput, instituía "infrações e crimes sanitários..." dispondo sobre "... processo administrativo para apuração de infrações sanitárias...".

Por ter sido essa proposta rechaçada pela maioria dos profissionais presentes naquela Oficina, em 2007, uma terceira minuta de Anteprojeto de Lei foi então apresentada, excluindo o SINVES e ficando restrito a "Emergências de Saúde Pública no Brasil". Todavia, dentre outras providências, além de estabelecer instrumentos de medidas de prevenção e controle das chamadas "Emergências de Saúde Pública" de relevância nacional, mantinha em seu caput a instituição de infrações e crimes sanitários. Entre os argumentos apresentados, a SVS/MS chamava a atenção para a necessidade do Brasil se preparar para implementar o RSI/2005, no que tangia à notificação à OPAS/ OMS de eventos de saúde considerados de relevância internacional. ${ }^{(28)}$

A divulgação dessa última proposta também provocou certo mal-estar e um acalorado debate envolvendo associações e instituições acadêmicas do campo da Saúde Coletiva, que, na sua maioria, se contrapuseram a este novo texto. As principais discordâncias manifestadas referem-se à inclusão de instruções sobre medidas restritivas da cidadania, inclusão de infrações sanitárias disciplinares e disposições penais, consideradas autoritárias, desnecessárias e que vão de encontro ao espírito democrático das Leis ns. $8.080 / 1990$ e $8.142 / 1990$. Tais desacordos estão apoiados nos dispositivos relativos aos direitos individuais garantidos na Carta Magna, pois a República Federativa do Brasil constitui-se em Estado Democrático de Direito sob os fundamentos da cidadania e da dignidade da pessoa humana, em que todos são iguais perante a lei, sem distinção de qualquer natureza. Portanto, está assegurada formalmente aos brasileiros e aos estrangeiros residentes no País a inviolabilidade do direito à vida, à liberdade, à igualdade, à segurança, dentre outras conquistas decorrentes das lutas pela redemocratização do país.

Considerando o que se encontra referido no inciso II do art. 5으 da Carta Constitucional brasileira, as leis restritivas de direitos, se realmente necessárias, devem ser cuidadosamente elaboradas, para que em nome "da necessidade do uso de força" para "... controle de riscos à saúde ...", atos arbitrários não venham a ser cometidos pela "autoridade de saúde" ainda que acompanhados da ressalva de que só serão adotados quando fundamentais e com base em informações sobre a eficácia, efetividade e segurança das mesmas ${ }^{(29)}$.

(28) BRASIL. Ministério da Saúde. Secretaria de Vigilância em Saúde. Anteprojeto de Lei. Emergências de saúde pública de relevância nacional: versão preliminar. Brasília: Ministério da Saúde, 2007. (29) BRASIL. Ministério da Saúde. Secretaria de Vigilância em Saúde. Anteprojeto de Lei. Emergências de saúde pública de relevância nacional: versão preliminar, cit. 
Tal concepção de Saúde Pública não pode ser simplesmente identificada com o Estado e promulgada em forma de lei, pois as leis têm como principal característica ser fenômeno social, ou seja, só existem em sociedade e não podem ser concebidas fora dela ${ }^{(30)}$. Já o campo científico da Saúde Coletiva que se tem desenvolvido em articulação com o processo da Reforma Sanitária Brasileira assenta-se em valores democráticos e emancipatórios, bem como no direito à saúde inerente à condição de cidadania. Por conseguinte, seus compromissos são com a população e, desse modo, a sua construção deve refletir os pactos com a sociedade.

\section{ALTERNATIVAS DE ATUAÇÃO FRENTE ÀS EMERGÊNCIAS DE SAÚDE PÚBLICA: MECANISMOS DE COERÇÃO SÃO NECESSÁRIOS?}

$\mathrm{Na}$ medida em que a população cresce e se desenvolve, o Direito tende a se aperfeiçoar, de forma a atender às necessidades de convivência, o que ocorre no interior de cada espaço nacional e no relacionamento entre as nações. Por essas e por outras razões, a lei deve representar o consenso comum e ter por finalidade essencial o interesse público, uma vez que tem por objetivo disciplinar as relações de interesse coletivo, com a finalidade de alcançar o bem-estar de todos.

Reconhece-se que no atual contexto do mundo globalizado, o fluxo de circulação de pessoas e mercadorias encontra-se em ritmo incontrolável, provocando desequilíbrio ambiental e potencializando os riscos e as consequências danosas para a vida humana, quase permanentemente, e influindo na circulação de agentes e vetores de doenças das mais variadas origens e potenciais de letalidade. Contudo, entende-se que não se justifica propor leis sanitárias que a priori sejam restritivas e coercitivas. Em nome do controle sanitário tais leis poderão afastar, ou até mesmo ignorar garantias estabelecidas na Constituição, instrumento norteador que baliza os direitos e deveres de todos os nacionais e estrangeiros presentes no território nacional, bem como os limites do próprio Estado. A supremacia dos princípios constitucionais, que são as verdades fundantes, os alicerces que, no Direito, se constituem nos pilares que sustentam o arcabouço jurídico, acenam para a garantia de segurança frente a arbítrios que possam levar a interpretações equivocadas de alguma norma jurídica gerando decisões danosas e ou constrangedoras.

Nessa perspectiva, fica evidente que as três versões de Projeto de Lei apresentadas por dirigentes e técnicos da área de vigilância e controle de doenças e agravos do Ministério da Saúde incluíram dispositivos que podem ser altamente invasivos à liberdade individual dos cidadãos e até violarem

(30) REALE, M. Lições preliminares de direito. São Paulo: Saraiva, 1977. 
direitos que asseguram a dignidade da pessoa humana. Não se pode olvidar o perigo que leis coercitivas das liberdades, mesmo que tenham como propósito a preservação da integridade da saúde da população ou de grupos de indivíduos, podem representar ameaças à democracia. Entende-se que, na dependência de uma determinada interpretação da Lei, torna-se mais difícil definir e limitar a atuação de agentes e órgãos encarregados pela execução de ações constrangedoras da liberdade de ir e vir, que venham compelir cidadãos à submissão a exames diagnósticos, imposição de tratamentos e isolamentos compulsórios, num país que mesmo declarado democrático e defensor dos direitos humanos, conforme arts. $1^{\circ}, 4^{\circ}$, II, art. $5^{\circ}$, dentre tantos outros da Constituição de 1988, ainda se encontra em fase de aprimoramento e consolidação da aplicação universal desses princípios em seu território.

Não seria aconselhável que as minutas de projetos de lei, até agora divulgadas, sejam encaminhadas para o Congresso Nacional, mesmo em nome de uma situação especial, tal como a possível vigência da pandemia de Influenza A. A sociedade precisa estar atenta e manter rigorosa cautela com a delegação de poder. É óbvio que em situações especiais como aquela referida, diversas medidas para a contenção do problema têm que ser adotadas, conforme já vem sendo feito pelas autoridades de saúde brasileiras, que em conjunto com outras instâncias da sociedade estão monitorando o problema, adotando as medidas pertinentes em acordo com o conhecimento técnico científico vigente e informando a população de forma clara e contínua com base em dados, análises e interpretações fidedignas. Têm-se utilizado mecanismos como a mobilização do Gabinete Permanente Interministerial criado pela Presidência da República, instituído para situações desta natureza, elaboração de Planos de Contingência Nacional e Estaduais, mobilização de recursos humanos, estruturas e da capacidade laboratorial instalada no país, busca de constante parceria e integração com os meios de comunicação para repasse das informações à sociedade, dentre outras iniciativas ${ }^{(31)}$. Assim, os dirigentes do SUS, nesta situação concreta a qual tem colocado em estado de alerta todo o globo terrestre, vêm trilhando o caminho do uso do saber técnico aliado à democratização das informações para cumprir seu mais elevado mister, qual seja, o de proteger a saúde da população sem lançar mão de qualquer gesto de autoritarismo.

Até o presente momento não se tem registro de resistência de cidadãos aos procedimentos individuais e coletivos adotados. O isolamento e o tratamento dos casos suspeitos de Influenza $A$ nos hospitais de referência do SUS ou da rede privada que dispõem de instalações preparadas para impedir a transmissão respiratória do vírus, estão sendo feitos em vários estados brasileiros sem necessidade do uso da força e da coerção. Ademais, caso

(31) BRASIL. Ministério da Saúde. Secretaria de Vigilância em Saúde. 2009. Disponível em: <http:// portal.saude.gov.br/portal/saude/profissional/area.cfm?id_area $=1534>$. 
haja alguma atitude isolada de resistência que não seja contornada pelos mecanismos de persuasão, e possa vir a exigir imposição da autoridade sanitária, as Leis brasileiras já contemplam dispositivos que podem ser acionados para resolver este tipo de ocorrência, a exemplo dos arts. 267, 268, 329 e 330 do Código Penal Brasileiro. ${ }^{(32)}$

A experiência que vem sendo acumulada mediante a implantação de estratégias para contenção dos mais de 500 eventos e que, de acordo com o $\mathrm{RSI} / 2005$, poderiam ser considerados "Emergências de saúde pública de importância nacional"(33) e as providências que estão sendo adotadas na atual situação de pandemia ${ }^{(34)}$ demonstram que o país não necessita de "leis de emergência" para adotar as medidas de Saúde Pública indicadas. Caso seja necessário, em razão de circunstâncias muito específicas, poder-se-á lançar mão de outros instrumentos normativos, como decretos, resoluções e portarias, indicando procedimentos pertinentes para cada tipo de doença e riscos. Em tais situações, seria preciso estabelecer, necessariamente vinculando a correspondente carga de responsabilidade às pessoas habilitadas para exercer as ações confiadas às denominadas "autoridades sanitárias"; prévio e rigoroso preparo e treinamento dos profissionais que atuarão na execução das ações sanitárias, não somente quanto às práticas de saúde, como também em relação à preservação máxima dos direitos e dignidade das pessoas integrantes da população alvo, respeitando as situações de possíveis fragilidades, vulnerabilidade e diversidade cultural, sem prejuízo das normas de saúde já vigentes no país.

$\mathrm{Na}$ medida em que se reconheça, oportunamente, ser imperativo a substituição da Lei n. 6.259/1975 por uma lei mais consentânea com o Estado Democrático de Direito e com os valores e princípios da Reforma Sanitária Brasileira e do SUS, além de uma adequação a novos perfis epidemiológicos e ao Regulamento Sanitário Internacional/2005, é defensável que os projetos elaborados se submetam ao mais amplo debate no âmbito da sociedade civil antes de serem encaminhados ao Congresso Nacional.

Tratando-se de tema tão candente, que tem provocado acirrado debate onde opiniões antagônicas se expressam, entende-se que, para se alcançar um consenso, alguns cuidados com o texto de uma nova Lei do SNVS devem ser tomados. Sendo este sistema subordinado ao SUS, por coerência, sua Lei tem que estar consonante com os objetivos e atribuições concernentes às vigilâncias incluídas no Capítulo I da Lei n. 8.080/90 e com o Decreto n. 4.726, 9 de junho de 2003 (no seu art. 20, Cap. I do ANEXO I da página 17 a 19). Mais

(32) BRASIL. Decreto-Lei n.2.848, de 7 de dezembro de 1940. Código Penal Brasileiro. Disponível em: <http://edutec.net/Leis/Gerais/cpb.htm>. Acesso em: 28 abr. 2009.

(33) CARMO, E. H.; PENNA, G.; OLIVEIRA, W. K. op. cit.

(34) BRASIL. Ministério da Saúde. Secretaria de Vigilância em Saúde. 2009. Disponível em: <http:// portal.saude.gov.br/portal/saude/profissional/area.cfm?id_area=1534>. 
que isso, é mandatório que respeite o "espírito" dessas duas legislações, pois este Decreto, ao aprovar a criação da SVS na nova estrutura regimental do Ministério da Saúde, definiu sua natureza e competências em estrita observância à Lei do SUS. No que se refere aos desdobramentos específicos relativos à Lei do SNVS, recomenda-se que estes devam ser direcionadas à formalização, consolidação e/ou fortalecimento dos progressos que vêm sendo obtidos no SUS desde 1991 quando da criação do CENEPI. Observe-se, que com maior ou menor grau de universalização e eficiência, alguns dos preceitos inscritos na Lei n. 8.080/1990 vêm sendo gradativamente incorporados ao SNVS atualmente em funcionamento. A descentralização da execução de ações, utilização de indicadores de avaliação, institucionalização da prática de uso da ferramenta epidemiológica no planejamento e na tomada de decisões, progressiva ampliação do escopo de atuação da vigilância epidemiológica (para doenças não transmissíveis, vigilância da mortalidade materna e infantil etc.); processo em curso de integração das vigilâncias (sanitária, epidemiológica, ambiental, saúde do trabalhador) nos três níveis de governo; integração com a atenção básica; mobilização e articulação dos serviços de saúde com as instituições de ensino e pesquisa do país são evidências dos avanços que vêm sendo alcançados nestes 20 anos do SUS. Ademais, entende-se que o campo de atuação da vigilância em saúde, por força da sua natureza que envolve controle de riscos, causas e danos; intervenções transetoriais organizadas que, no mais das vezes, exigem intensa e contínua participação social, representa espaço privilegiado para impulsionar, dentro do SUS, o processo de desenvolvimento da Promoção da Saúde.

Nessa perspectiva, recomenda-se que uma nova minuta de Lei para o SNVS deva atender a expectativa, que permeia mentes e energias de parcelas significativas da comunidade que milita no campo da saúde coletiva, que tem como horizonte um Brasil caminhando, cada vez mais, para a implantação da Reforma Sanitária arquitetada na histórica VIII Conferência Nacional de Saúde. Assim o texto, deve conter conceitos, concepções, definições de competências e atribuições, ordenação de operações e ações que venham a contribuir substantivamente para o alcance desta imagem objetivo.

\section{REFERÊNCIAS BIBLIOGRÁFICAS}

BARATA, R. C. B. O desafio das doenças emergentes e a revalorizaçäo da epidemiologia descritiva. Revista de Saúde Pública, São Paulo, v. 31, n. 5, p. 531-537, 1997.

BARRETO, M. L. Determinantes das condições de saúde e problemas prioritários do país. In: Conferência Nacional de Saúde, 11. Caderno... Brasília: Ministério da Saúde; 2000. p. 235-259. 
BRASIL. Constituição da República Federativa do Brasil: promulgada em 5 de outubro 1988. São Paulo. Saraiva, 2000.

Decreto n. 78.231 de 12 de agosto de 1976. Regulamenta a Lei n. $\overline{6.259}$, de 30 e outubro de 1975, que dispõe sobre a organização das ações de Vigilância Epidemiológica, sobre o Programa Nacional de Imunizações, estabelece normas relativas à notificação compulsória de doenças, e dá outras providências. Disponível em: <http://www.aids.gov.br/c-geral/ Ic0303.htm>. Acesso em: 15 abr. 2009.

Decreto-Lei n. 2.848, de 7 de dezembro de 1940. Código Penal Brasileiro. Disponível em: <http://edutec.net/Leis/Gerais/cpb.htm>. Acesso em: 28 abr. 2009.

. Ministério da Saúde. Conferência Nacional de Saúde, 11. Caderno ... Brasília: Ministério da Saúde; 2000. 336 p.

. Ministério da Saúde. Lei n. 8.142 de 28 de dezembro de 1990. Dispõe sobre a participação da comunidade na gestão do Sistema Único de Saúde - SUS e sobre as transferências intergovernamentais de recursos financeiros na área da saúde e dá outras providências. Brasília: Ministério da Saúde, 1990.

. Ministério da Saúde. Lei n. 8080 de 19 de setembro de 1990: dispõe sobre as condições para promoção, proteção e recuperação da saúde, a organização e o funcionamento dos serviços correspondentes e dá outras providências. Brasília: Ministério da Saúde, 1990. Disponível em: <http:// www6.senado.gov.br/legislacao/ListaPublicacoes.action?id=134238>. Acesso em: 30 abr. 2009.

. Ministério da Saúde. Secretaria de Vigilância em Saúde. 2009. Disponível em: <http://portal.saude.gov.br/portal/saude/profissional/ area.cfm?id_area $=1534>$.

Ministério da Saúde. Secretaria de Vigilância em Saúde. Anteprojeto de Lei. Emergências de saúde pública de relevância nacional: versão preliminar. Brasília: Ministério da Saúde, 2007.

. Ministério da Saúde. Secretaria de Vigilância em Saúde. Projeto de Lei do Sistema Nacional de Vigilância em Saúde. Brasília: SVS/CEPEDISA/ USP, 2006. (Texto para uso interno na oficina da 6 $6^{\underline{a}}$ EXPOEPI, realizada em Brasília em 14 nov. 2006). Disponível em: <http://www.anerbrasil.org.br/sites/700/797/00000101.doc.>. Acesso em: 6 maio 2009.

Projeto de Lei. Dispõe sobre os Sistemas Nacionais de Epidemiologia, de Saúde Ambiental e de Saúde Indígena, cria a Agência Federal de Prevenção e Controle de Doenças - APEC, e dá outras providências. 2002. Disponível em: <www.camara.gov.br/sileg/Mostrarlntegra. asp?CodTeor=49706>. Acesso em: 15 abr. 2009. 
CARMO, E. H.; BARRETO, M. L.; SILVA JUNIOR, J. B. Mudanças nos padrões de morbimortalidade da população brasileira: os desafios para um novo século. Epidemiol. Serv. Saúde, v. 12, n. 2, p. 63-75, abr./jun. 2003.

; PENNA, G.; OLIVEIRA, W. K. Emergências de saúde pública: conceito, caracterização, preparação e resposta. Estudos Avançados, São Paulo, v. 22, n. 64, p. 19-32, 2008.

COSTA, E. A. Vigilância sanitária, defesa e proteção da saúde. 1998.350 p. Tese (Doutorado) - Faculdade de Saúde Pública, Universidade de São Paulo, 1998.

COSTA, Nilson do Rosário. Lutas urbanas e controle sanitário: origens das políticas de saúde no Brasil. Rio de Janeiro: Vozes, 1985.

EDITORIAL. Outra emenda pior que o soneto: APEC - novas ameaças ao processo de descentralização da saúde. Bol Abrasco, n. 84, 2002.

ESCOREL, S.; TEIXEIRA, L. A. História das políticas de saúde no Brasil de 1822 a 1963: do império ao desenvolvimento populista. In: GIOVANELLA, L. (Org.). Políticas e sistemas de saúde no Brasil. Rio de Janeiro: Fiocruz, 2008. p. 333-384.

FOSSAERT, D. H.; LLOPIS, A.; TIGRE, C. H. Sistemas de vigilância epidemiológica. Bol Ofic Sanit Panam., n. 76, p. 512-525, 1974.

GUBLER, D. J. Dengue and dengue hemorrhagic fever. Clin. Microbiol. Rev., v. 11 , n. 3, p. 480-496, 1998.

LANGMUIR, A. D. The surveillance of communicable diseases of national importance. New Engl. J. Med., n. 268, p. 182-192, 1963.

OOI, E. E.; GOH, K.T.; GUBLER, D. J. Dengue Prevention and 35 Years of Vector Control in Singapore. Emerg. Infect. Dis., v. 12, n. 6, p. 887-893, 2006.

ORGANIZAÇÃO MUNDIAL DA SAÚDE. Regulamento Sanitário Internacional, 1969. 3. ed. anot. Ginebra: OMS, 1983.

PAIM, J. S.; TEIXEIRA, M. G. Reorganização do sistema de vigilância epidemiológica na perspectiva do Sistema Único de Saúde - SUS. Inf. Epidemiol, SUS, v. 1, n. 5, p. 27-57, out. 1992.

RASKA, K. National and international surveillance of communicable diseases. Who Chron, n. 20, p. 31-21, 1966.

REALE, M. Lições preliminares de direito. São Paulo: Saraiva, 1977. 430p.

TEIXEIRA, C. F.; PAIM, J. S.; VILASBÔAS, A. L. SUS, modelos assistenciais e vigilância da saúde. Inf. Epidemiol. SUS, v. 7, n. 2, p. 7-28, abr./jun. 1998.

TEIXEIRA, M. G. Editorial especial: Secretaria de Vigilância em Saúde: novas perspectivas para o SUS. Rev. bras. epidemiol., v. 6, n. 3, p. 197-199, 2003. 
; COSTA, M. C. N. Vigilância epidemiológica: políticas, sistemas e serviços. In: GIOVANELLA, L. (Org.). Políticas e sistemas de saúde no Brasil. Rio de Janeiro: Fiocruz, 2008. p. 795-817.

; BARRETO, F. Dengue: twenty-five years since reemergence in Brazil. Cad. Saúde Pública, v. 25, suppl.1, p. S7-S18, 2009.

; RISI JÚNIOR, J. B.; COSTA, M. C. N. Vigilância epidemiológica. In: ROUQUAYROL, M. Z.; ALMEIDA FILHO, N. Epidemiologia e saúde. 6. ed. Rio de Janeiro: Medsi, 2003. p. 313-356.

WORLD HEALTH ASSEMBLY. Revision of the International Health Regulations, WHA 58.3. 2005. Disponível em: <http://www.who.int>. Acesso em: 10 maio 2009. 